\title{
Cancer Risk in Pulmonary Hypertension Patients
}

\author{
Henrik Toft Sørensen (D)', Nils Skajaa', Frederikus Albertus Klok², Kristina Laugesen (iD', \\ Dóra Körmendiné Farkas' \\ 'Department of Clinical Epidemiology, Aarhus University Hospital and Aarhus University, Aarhus N, DK-8200, Denmark; ${ }^{2}$ Department of Medicine -
Thrombosis and Hemostasis, Leiden University Medical Center, Leiden, the Netherlands
}

Correspondence: Henrik Toft Sørensen, Department of Clinical Epidemiology, Aarhus University, Olof Palmes Allé 43-45, Aarhus N, DK-8200, Denmark, Email hts@clin.au.dk

Background: Strong evidence indicates that venous thromboembolism is a presenting symptom of cancer. Cancer is a known cause of pulmonary hypertension; however, it remains unknown whether pulmonary hypertension is a marker of occult cancer. We examined the association between a pulmonary hypertension diagnosis and cancer risk in a cohort study using population-based data from the Danish health system.

Patients and Methods: Using Danish nationwide registries, we identified 6335 patients with a pulmonary hypertension diagnosis and without a previous cancer diagnosis between 1995 and 2017. We computed the age-, sex-, and calendar year-standardized incidence ratio (SIR) as the ratio of observed to expected number of cancers using national incidence rates as the reference. We performed a subgroup analysis among patients with chronic thromboembolic pulmonary hypertension in the period in which a specific ICD-10 code was available (2006-2017).

Results: We identified 212 cancers within the first year of follow-up and 796 cancers thereafter. The one-year risk of cancer was 3.3\% and the one-year SIR was 1.96 (95\% confidence interval [CI]: 1.70-2.23). In the second and subsequent years, the SIR remained elevated (SIR: 1.15 [95\% CI: 1.08-1.24]). In patients with chronic thromboembolic pulmonary hypertension, the one-year SIR was 1.41 (95\% CI: 0.82-2.25).

Conclusion: Cancer risk was clearly higher in patients with pulmonary hypertension compared with the general population. The association was particularly strong in the first year of follow-up, but remained elevated thereafter. However, absolute risks were low, limiting the clinical relevance of pursuing early cancer detection in these patients.

Keywords: pulmonary hypertension, chronic thromboembolic pulmonary hypertension, venous thrombosis, pulmonary embolism, cancer, cohort

\section{Introduction}

Pulmonary arterial hypertension (PAH; WHO group 1 pulmonary hypertension) is a rare but serious condition, characterized by elevated pulmonary artery pressure and contributing to increased morbidity and mortality. ${ }^{1}$ Pulmonary hypertension ( $\mathrm{PH})$ may be idiopathic or secondary to several chronic diseases, including left-sided heart disease, chronic obstructive pulmonary disease, collagen vascular disease, and venous thromboembolism (VTE). ${ }^{1}$

VTE is an established risk factor for chronic thromboembolic pulmonary hypertension (CTEPH; WHO group $4 \mathrm{PH}){ }^{2}$ although CTEPH occurs in patients with no history of symptomatic VTE in $19 \%$ to $63 \%$ of cases. ${ }^{3}$ Such patients may have suffered from asymptomatic VTE, but this remains unknown. An association between cancer and VTE has been recognized for at least 150 years. ${ }^{4}$ During recent decades, strong evidence has accumulated indicating that VTE may be a presenting symptom of cancer.,

Similarly, exposure to carcinogenic substances, such as smoking and occupational hazards, is associated with group 3 and group $5 \mathrm{PH}$. Further, cancer itself - or its treatment - is known to cause pulmonary hypertension, particularly pulmonary tumor thrombotic microangiopathy and pulmonary veno-occlusive disease. ${ }^{1}$ Hence it may be hypothesized that PH, like VTE, could be a marker of occult cancer. A small French cohort study of 346 patients with group 1, group 3 , or group $4 \mathrm{PH}$ found that 5 -year cancer incidence approached $15 \% .{ }^{6}$ Although this knowledge is very relevant for the 
management of $\mathrm{PH}$ patients, the association of cancer with $\mathrm{PH}$ compared to the general population is unknown. We therefore examined the association between a PH diagnosis and cancer risk in a cohort study using population-based data from the Danish health system.

\section{Methods}

The study was based on the cumulative Danish population of 7.6m persons during the 1995-2017 period. Danish residents have free access to tax-supported health care. ${ }^{7}$ We used the Danish National Patient Registry and the Danish Cancer Registry, which cover all Danish hospitals, to examine cancer incidence in 6335 patients with PH and without a previous cancer diagnosis. The civil registration number unique to every Danish citizen allowed linkage between the registries. $^{7}$ Diseases in the registries are classified according to the International Classification of Diseases, Tenth Revision (ICD-10; ICD-8 codes were used before 1994). The study setting, registries, and variable definitions are described in detail in the Supplemental Material. We considered the date of first hospital admission or start of outpatient clinic follow-up to be the PH diagnosis date. We had access only to PH diagnoses based on ICD codes (Table S1). As a result, we lacked information on diagnostic criteria, severity, or subgroups of pulmonary hypertension, except for CTEPH, during part of the study period.

All the patients were followed from the PH diagnosis date until death or December 31, 2018, whichever came first. We calculated the expected number of cancer cases after the first hospital contact for $\mathrm{PH}$, based on national incidence rates of first cancer diagnoses according to sex, age, and year of diagnosis in 1-year intervals. Multiplication of the number of person-years of follow-up by national incidence rates yielded the number of people with cancer that would be expected if patients with $\mathrm{PH}$ had the same risk of cancer as the general population. We used the standardized incidence ratio (SIR) - the ratio of observed number of cancers to expected number of cancers - to measure the association between $\mathrm{PH}$ and cancer, and calculated $95 \%$ confidence intervals (CIs). In the setting of a PH diagnosis, early cancer detection likely captures malignancies that would otherwise remain occult. For this reason, we examined the association with cancer according to arbitrarily chosen time periods since a PH diagnosis ( $\leq 1$ year, $>1$ year). We performed a subgroup analysis for the period during which a specific ICD-10 code was available for CTEPH (2006-2017).

Treating death as a competing risk, we calculated the one-year risk of cancer following a PH diagnosis. Assuming that cancers detected during the first year of follow-up were present at the time of diagnosis, we computed the number of patients needing to be examined at the time of $\mathrm{PH}$ diagnosis in order to detect one excess cancer, as the reciprocal of the excess cancer risk.

We performed the analyses in SAS. The study was reported to the Danish Data Protection Agency (consent from patients is not required for Danish registry-based studies).

\section{Results}

We followed the 6335 patients for a median of 4.1 years. Table 1 provides descriptive data on the study cohort. During the first year of follow-up, 212 cancers were diagnosed. After the first year of follow-up, 796 cancer cases were diagnosed. The one-year risk was 3.3\% and the one-year SIR was 1.96 (95\% CI: 1.70-2.24). We found an elevated risk of several types of cancers (Figure 1). The cancer risk remained elevated even after one year of follow-up (SIR: 1.15 (95\% CI: 1.08-1.24). The one-year SIR for patients with CTEPH was 1.41 (95\% CI: 0.82-2.25). The number of patients with $\mathrm{PH}$ needing to be examined to detect one excess cancer was 52.

\section{Discussion}

Cancer risk was clearly higher in patients with $\mathrm{PH}$ compared with the general population. We found a dichotomous pattern of cancer risk over time. In the first year after a PH diagnosis, cancer risk was particularly elevated. However, PH also was associated with long-term cancer risk. The same pattern is well established for the association between VTE and cancer. ${ }^{4,5}$

Cancer may be associated with PH for several reasons. Heightened diagnostic efforts and the effects of occult cancer probably explain the short-term association, but we did not see a compensatory decreased incidence after the first year, characteristic for such a pattern. Other explanations for the persistent association are therefore required. 
Table I Characteristics of the Study Cohort of Patients with Pulmonary Hypertension, Denmark, 1995-2017

\begin{tabular}{|c|c|c|c|c|}
\hline \multirow[t]{2}{*}{ Variable } & \multicolumn{2}{|c|}{ Pulmonary Hypertension } & \multicolumn{2}{|c|}{$\begin{array}{l}\text { Sub-Cohort of Patients with a } \\
\text { Specific CTEPH Diagnosis }\end{array}$} \\
\hline & $\mathbf{N}$ & $\%$ & $\mathbf{N}$ & $\%$ \\
\hline \multicolumn{5}{|l|}{ Gender } \\
\hline Male & 2847 & 44.9 & 276 & 45.7 \\
\hline Female & 3488 & 55.1 & 328 & 54.3 \\
\hline \multicolumn{5}{|l|}{ Age, years } \\
\hline $0-54$ & 1420 & 22.4 & 123 & 20.4 \\
\hline $55-64$ & 1199 & 18.9 & 98 & 16.2 \\
\hline $65-74$ & 1686 & 26.6 & 170 & 28.1 \\
\hline $75+$ & 2030 & 32.0 & 213 & 35.3 \\
\hline \multicolumn{5}{|l|}{ Calendar period } \\
\hline $1995-1999$ & 968 & 15.3 & - & - \\
\hline $2000-2004$ & 1398 & 22.1 & - & - \\
\hline $2005-2009$ & 1665 & 26.3 & 97 & 16.1 \\
\hline $2010-2014$ & 1357 & 21.4 & 325 & 53.8 \\
\hline $2015-2017$ & 947 & 14.9 & 182 & 30.1 \\
\hline \multicolumn{5}{|l|}{ Hypothyroidism } \\
\hline No & 6172 & 97.4 & 577 & 95.5 \\
\hline Yes & 163 & 2.6 & 27 & 4.5 \\
\hline \multicolumn{5}{|c|}{ Hyperthyroidism } \\
\hline No & 6098 & 96.3 & 590 & 97.7 \\
\hline Yes & 237 & 3.7 & 14 & 2.3 \\
\hline \multicolumn{5}{|c|}{ Venous thromboembolism } \\
\hline No & 5770 & 91.1 & 207 & 34.3 \\
\hline Yes & 565 & 8.9 & 397 & 65.7 \\
\hline \multicolumn{5}{|c|}{ Pulmonary embolism \pm deep venous thrombosis } \\
\hline No & 5910 & 93.3 & 222 & 36.8 \\
\hline Yes & 425 & 6.7 & 382 & 63.2 \\
\hline \multicolumn{5}{|c|}{ Deep venous thrombosis } \\
\hline No & 6195 & 97.8 & 589 & 97.5 \\
\hline Yes & 140 & 2.2 & 15 & 2.5 \\
\hline \multicolumn{5}{|c|}{ Chronic obstructive pulmonary disease } \\
\hline No & 5010 & 79.1 & 433 & 71.7 \\
\hline Yes & 1325 & 20.9 & 171 & 28.3 \\
\hline \multicolumn{5}{|c|}{ Interstitial lung disease } \\
\hline No & 5941 & 93.8 & 553 & 91.6 \\
\hline Yes & 394 & 6.2 & 51 & 8.4 \\
\hline \multicolumn{5}{|l|}{ HIV } \\
\hline No & 6316 & 99.7 & 604 & 100 \\
\hline Yes & 19 & 0.3 & 0 & 0 \\
\hline \multicolumn{5}{|c|}{ Portal hypertension } \\
\hline No & 6306 & 99.5 & - & - \\
\hline Yes & 29 & 0.5 & $<5^{\mathrm{a}}$ & - \\
\hline \multicolumn{5}{|l|}{ Splenectomy } \\
\hline No & 6317 & 99.7 & 593 & 98.2 \\
\hline Yes & 18 & 0.3 & 11 & 1.8 \\
\hline \multicolumn{5}{|c|}{ Connective tissue disorders } \\
\hline No & 5789 & 91.4 & 549 & 90.9 \\
\hline Yes & 546 & 8.6 & 55 & 9.1 \\
\hline \multicolumn{5}{|l|}{ Heart failure } \\
\hline No & 5185 & 81.8 & 481 & 79.6 \\
\hline Yes & 1150 & 18.2 & 123 & 20.4 \\
\hline Total & 6335 & 100 & 604 & 100 \\
\hline
\end{tabular}

Notes: ${ }^{a}$ According to Danish privacy rules, no cells with fewer than 5 observations may be reported.

Abbreviation: CTEPH, chronic thromboembolic pulmonary hypertension. 

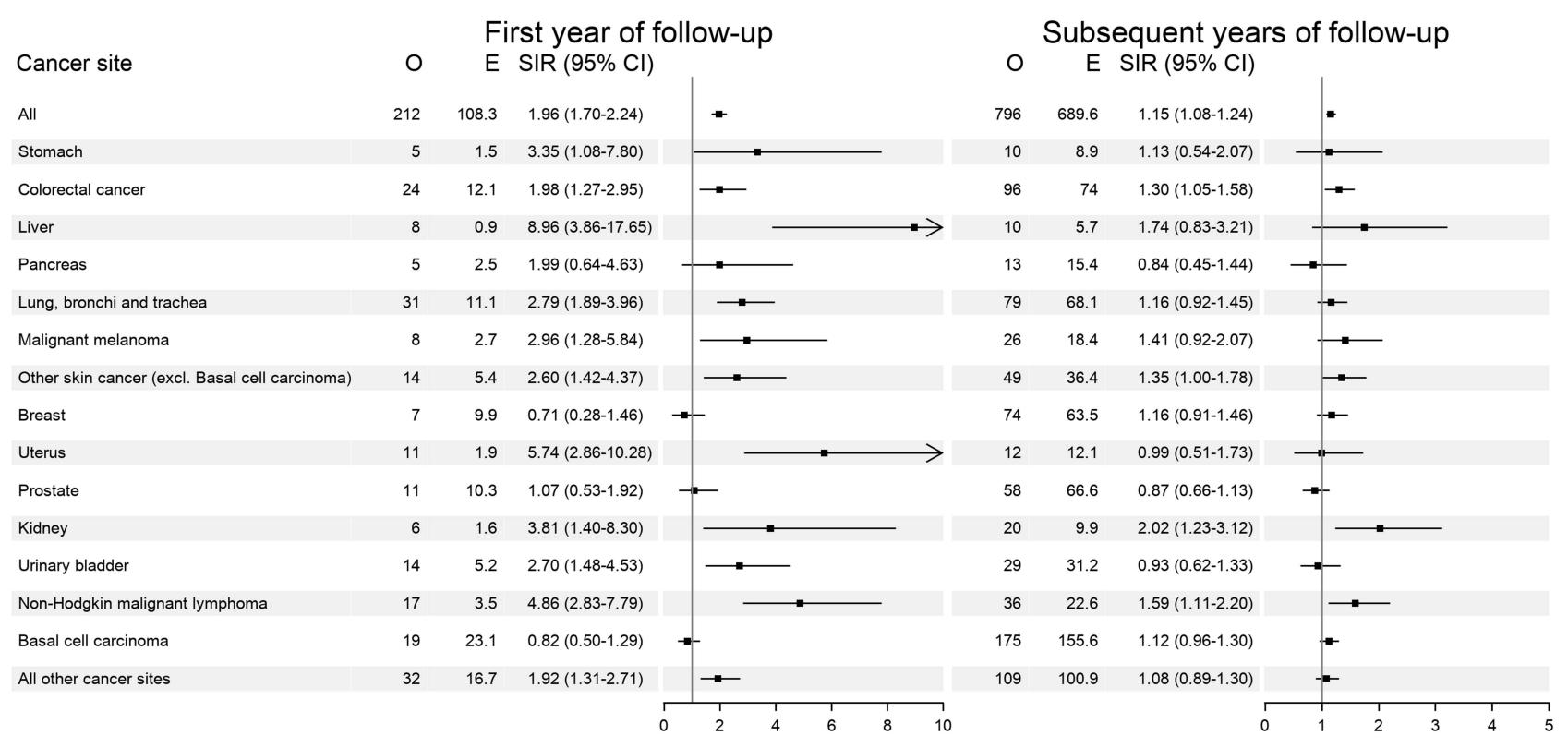

Figure I Standardized Cancer Incidence Ratios for Patients with Pulmonary Hypertension.

Abbreviations: $\mathrm{O}$, observed number of cancer cases; $\mathrm{E}$, expected number of cancer cases; SIR, standardized incidence ratio; $\mathrm{Cl}$, confidence interval.

In summary, we identified an increased incidence of cancer in PH patients, although the absolute risks were low and the number needed to examine was high, limiting the clinical relevance of pursuing early cancer detection in these patients. Moreover, although our population-based cohort study was large and had virtually no loss to follow-up, our data lacked clinical detail and relied on ICD diagnoses. The high number needed to examine and these limitations prevent specific guidelines for cancer work-up in patients with different types of pulmonary hypertension and should be based on the clinical findings.

\section{Ethics Statement}

According to Danish law, studies based entirely on registry data do not require approval from an ethics review board. This study was reported to the Danish Data Protection Agency through registration at Aarhus University (record No KEA-2017-36/812).

\section{Author Contributions}

All authors made a significant contribution to the work reported, whether that is in the conception, study design, execution, acquisition of data, analysis and interpretation, or in all these areas; took part in drafting, revising or critically reviewing the article; gave final approval of the version to be published; have agreed on the journal to which the article has been submitted; and agree to be accountable for all aspects of the work.

\section{Funding}

There is no funding to report.

\section{Disclosure}

The authors declare no conflicts of interest in this work. 


\section{References}

1. Galiè N, Humbert M, Vachiery JL, et al. 2015 ESC/ERS Guidelines for the diagnosis and treatment of pulmonary hypertension: the joint task force for the diagnosis and treatment of pulmonary hypertension of the European Society of Cardiology (ESC) and the European Respiratory Society (ERS): endorsed by: Association for European Paediatric and Congenital Cardiology (AEPC), International Society for Heart and Lung Transplantation (ISHLT). Eur Heart J. 2016;37(1):67-119.

2. Klok FA, Delcroix M, Bogaard HJ. Chronic thromboembolic pulmonary hypertension from the perspective of patients with pulmonary embolism. $J$ Thromb Haemost. 2018;16(6):1040-1051. doi:10.1111/jth.14016

3. Pepke-Zaba J, Delcroix M, Lang I, et al. Chronic thromboembolic pulmonary hypertension (CTEPH): results from an international prospective registry. Circulation. 2011;124(18):1973-1981. doi:10.1161/CIRCULATIONAHA.110.015008

4. Sørensen HT, Mellemkjaer L, Steffensen FH, Olsen JH, Nielsen GL. The risk of a diagnosis of cancer after primary deep venous thrombosis or pulmonary embolism. $N$ Engl J Med. 1998;338(17):1169-1173. doi:10.1056/NEJM199804233381701

5. Baron JA, Gridley G, Weiderpass E, Nyren O, Linet M. Venous thromboembolism and cancer. Lancet. 1998;351(9109):1077-1080.

6. Bravos E, Cottin V, Dauphin C, et al. Cancer incidence in patients with pre-capillary pulmonary hypertension. J Heart Lung Transplant. 2019;38 (7):778-780. doi:10.1016/j.healun.2019.03.007

7. Schmidt M, Schmidt SAJ, Adelborg K, et al. The Danish health care system and epidemiological research: from health care contacts to database records. Clin Epidemiol. 2019;11:563-591. doi:10.2147/CLEP.S179083

Clinical Epidemiology

\section{Publish your work in this journal}

Clinical Epidemiology is an international, peer-reviewed, open access, online journal focusing on disease and drug epidemiology, identification of risk factors and screening procedures to develop optimal preventative initiatives and programs. Specific topics include: diagnosis, prognosis, treatment, screening, prevention, risk factor modification, systematic reviews, risk \& safety of medical interventions, epidemiology \& biostatistical methods, and evaluation of guidelines, translational medicine, health policies \& economic evaluations. The manuscript management system is completely online and includes a very quick and fair peer-review system, which is all easy to use.

Submit your manuscript here: https://www.dovepress.com/clinical-epidemiology-journal 